\title{
More on an Erdós-Szekeres-Type Problem for Interior Points
}

\author{
Xianglin Wei · Ren Ding
}

Received: 7 December 2007 / Revised: 22 April 2008 / Accepted: 25 May 2008 /

Published online: 20 June 2008

(C) Springer Science+Business Media, LLC 2008

\begin{abstract}
An interior point of a finite planar point set is a point of the set that is not on the boundary of the convex hull of the set. For any integer $k \geq 1$, let $g(k)$ be the smallest integer such that every planar point set in general position with at least $g(k)$ interior points has a convex subset of points with exactly $k$ interior points of $P$. In this article, we prove that $g(3)=9$.
\end{abstract}

Keywords Interior point - General position - Empty convex polygon - Splitter · Deficient point set

\section{Introduction}

In this article, we consider only point sets in the plane. All points are assumed to be in general position, that is, no three of the points are collinear. The most famous and attractive problem concerning finite point sets is the following Erdôs-Szekeres Problem posed in 1935 (see [5-7, 13]): For any integer $n \geq 3$, determine the smallest positive integer $N(n)$ such that any set of at least $N(n)$ points contains $n$ points that are the vertices of a convex $n$-gon. In their first joint paper [6], Erdős and Szekeres proved the existence of the number $N(n)$ by two different methods. In [7] they obtained the lower bound $N(n) \geq 2^{n-2}+1$ for all $n \geq 3$. $N(3)=3$ is trivial. The only known nontrivial exact values of $N(n)$ are $N(4)=5$ (see [4]) and $N(5)=9$

This research was supported by National Natural Science Foundation of China (10571042, 10701033), NSF of Hebei (A2005000144, A2007000226, A2007000002) and the SF of Hebei Normal University (L2004202).

X. Wei

College of Science, Hebei University of Science and Technology, Shijiazhuang 050018, China e-mail: wxlhebtu@126.com

X. Wei $\cdot$ R. Ding $(\bowtie)$

College of Mathematics, Hebei Normal University, Shijiazhuang 050016, China

e-mail: rending@hebtu.edu.cn 
(see [11]). For generalizations of Erdős-Szekeres Problem to families of convex sets, see [14] and [15].

In 2001, Avis, Hosono, and Urabe [2] investigated the following question: For any integer $k \geq 1$, determine the smallest integer $g(k)$ such that every set of points containing at least $g(k)$ interior points has a subset containing exactly $k$ interior points.

In [2] the authors showed that $g(1)=1, g(2)=4$, and $g(3) \geq 8$ and also that every point set with at least $k$ interior points contains a subset with between $k$ and $\left\lfloor\frac{3 k}{2}\right\rfloor$ interior points, and every point set with at least 3 interior points contains a subset with either 3 or 4 interior points. In 2005, Hosono [9] presented the following result: if a point set has at least 8 interior points and no empty convex hexagons, then it contains a subset with precisely 3 interior points. The progress in the investigation of the lower bound of $g(k)$ for integer $k$ is as follows: In [2] it is proved that $g(k) \geq k+2$ for $k \geq 4$. In [1] it is proved that $g(k) \geq\lceil(3 k+3) / 2\rceil$ for $k \geq 6$. Fevens [8] showed that $g(k) \geq 3 k-1$ for $k \geq 3$. Recently, we improved the lower bound by proving that $g(k) \geq 3 k$ for $k \geq 3$ (see [17]).

The existence or finiteness of $g(k)$ for any nonnegative integer $k$ is still an open problem. Let $g_{\triangle}(k)$ be the smallest integer such that every set of points whose convex hull is a triangle and which has at least $g_{\triangle}(k)$ interior points also contains a subset with exactly $k$ interior points. By using some results from [12] and [16], Hosono et al. [10] and Bisztriczky et al. [3] proved that if $g_{\triangle}(k)$ is finite, $g(k)$ is also finite for every nonnegative integer $k$.

In this paper, we discuss the existence of a point subset with exactly 3 interior points of $P$ and obtain that $g(3)=9$.

\section{Definitions and Notation}

Let $P$ be a finite planar point set in general position. Let $C H(P)$ denote the convex hull of $P$, and $V(P)=\left\{v_{1}, v_{2}, \ldots, v_{m}\right\}$ be the vertex set of $C H(P)$, or briefly, of $P$. Here the vertices $v_{1}, v_{2}, \ldots, v_{m}$ are always in counter-clockwise order. An interior point of $P$ is a point of $P$ that is not on the boundary of $C H(P)$. Let $I(P)$ denote the interior point set of $P$. Let $Q \subset P$ be a subset of $P$; we say that $Q$ contains an interior point $p$ of $P$ if $p \in I(P) \cap \operatorname{intCH}(Q)$, where $\operatorname{intCH}(Q)$ is the interior of the convex hull of $Q$, and we shorten $I(P) \cap \operatorname{int} C H(Q)$ to $I^{*}(Q)$. It is easy to give examples showing that $I(Q) \neq I^{*}(Q)$. Denote by $i^{*}(Q)=\left|I^{*}(Q)\right|$ the number of interior points of $P$ contained in $Q$. When $i^{*}(Q)=k$, we say that $Q$ is a "subset with exactly $k$ interior points of $P$," or a "subset with exactly $k$ interior points" for brevity. If a point set $R$ (a connected region or a finite point set in the plane) contains no interior point of $P$, we say that $R$ is empty, which is denoted by $R \approx \emptyset$.

Using the above notation, $g(k)$ can be defined as follows:

$$
g(k)=\min \left\{s:|I(P)| \geq s \Rightarrow \exists Q \subset P \text { such that } i^{*}(Q)=|I(P) \cap \operatorname{int} C H(Q)|=k\right\} .
$$

A point set $T$ is called a triangle and denoted by $T=\triangle v_{1} v_{2} v_{3}$ if $V(T)=$ $\left\{v_{1}, v_{2}, v_{3}\right\}$. An interior point of a triangle $T$ is called an $(x, y, z)$-splitter, or a splitter of type $(x, y, z)$ of $T$ if it partitions $T$ into three triangles with $x \geq y \geq z$ interior 
points, respectively. In the case $z=0$, we abbreviate it to $(x, y)$-splitter. Let $H(a b ; c)$ denote the half plane bounded by the straight line $a b$ with $c$ in the interior of the half plane, and $C(a ; b, c)$ a convex cone, where $a$ is the apex of the cone and the two rays $a b$ and $a c$ form the boundary of the cone. We say that a point set $Q$ is in convex position, or convex, when it forms the vertex set of a point set.

To investigate the lower bounds for $g(k)$, we need the concept of deficient point sets (see [2]). A point set $P$ is called a deficient point set of type $P(m, s, k)$ and denoted as $P=P(m, s, k)$ if $|V(P)|=m,|I(P)|=s$, and for any $Q \subset P$, $i^{*}(Q) \neq k$. For brevity, we may use $P(m, s, k)$ to stand for the deficient set itself. The existence of a deficient point set $P=P(m, s, k)$ implies that $g(k) \geq s+1$.

For a point set $P$, an edge of $C H(P)$ is also called an edge of $P$. If for an edge $x y$ of $P(3, s, 3)$, there exists a subset $Q \subset P$ with $i^{*}(Q)=k$ such that $x y$ is an edge of $Q$, we say the edge $x y$ is of type- $k$, where $k \neq 3$ and $k \leq s-1$.

We call a subset $Q$ of $P$ a 3 -int subset when $i^{*}(Q)=3$, that is, when $Q$ contains exactly 3 interior points of $P$.

According to [1], a configuration of a deficient point set $P$ with certain required properties is called a monster.

\section{Basic Lemmas}

For the proof of $g(3)=9$, we need the following properties of deficient point sets of type $P(3, s, 3)$ with $4 \leq s \leq 8$, which are proved in [18].

Lemma 1 [18] A deficient point set $P=P(3,4,3)$ has the following properties:

1. Every edge of $P$ is of type-1 or of type-2;

2. $P$ has at least two edges each of which is of both type-1 and type-2.

$P(3,5,3)$ has the same properties.

Lemma 2 [18] Every edge of a deficient point set $P(3,6,3)$ is of both type-1 and type-2. $P(3,7,3)$ and $P(3,8,3)$ have the same property.

In the proof of Lemma 1 , we obtain two different configurations of $P(3,5,3)$ called a 5-I-monster and a 5-II-monster and shown in Fig. 1. In a 5-I-monster, one

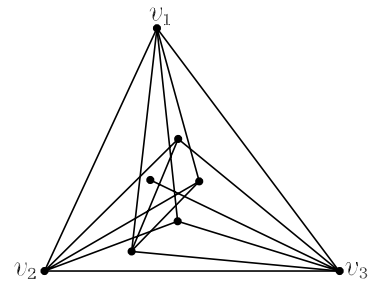

Fig. 1 (a) 5-I-monster; (b) 5-II-monster (b)

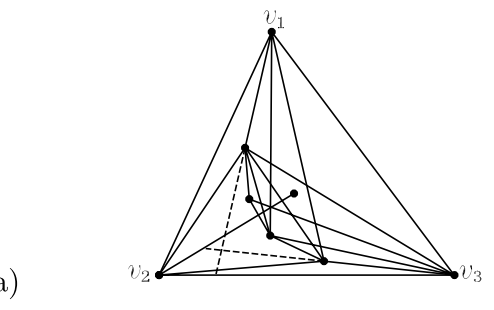

(a) 
edge $v_{1} v_{3}$ is of type-2 but not of type- 1 (see Fig. 1a). In a 5-II-monster, one edge $v_{1} v_{3}$ is of type-1 but not of type-2 (see Fig. 1b).

In the proof of Lemma 2, we obtain three different configurations of $P(3,8,3)$ called an 8-I-monster, an 8-II-monster, and an 8-III-monster, respectively.

We also need the following lemma.

Reduction Lemma [2] Let $P$ be a planar point set with $m \geq 3$ vertices and $s \geq 0$ interior points. There exists a vertex $v_{i}$ of $P$ such that $P^{\prime}=P \backslash\left\{v_{i}\right\}$ has $m^{\prime}$ vertices, $s^{\prime}$ interior points, and either $(a) m^{\prime}=m-1$ and $s^{\prime}=s$ or $(b) m^{\prime}=m+t$ and $s^{\prime}=s-t-1$, where $t=0,1,2, \ldots,\lfloor s / m\rfloor$.

\section{The Main Result and Its Proof}

Theorem $g(3)=9$.

The existence of $P(3,8,3)$ (see Fig. 2) shows that $g(3) \geq 9$. Therefore, to prove that $g(3)=9$, it suffices to prove that $g(3) \leq 9$.

According to the definition of $g(3)$, what we need to prove is that for any point set $P$ with $|I(P)| \geq 9$, there always exists a 3-int subset of $P$.

We split our conclusion into several lemmas. For brevity, in the proofs of the following lemmas, we always assume that every triangle $\triangle$ with $i^{*}(\triangle)=4,5,6$, 7, or 8 is a deficient point set (or monster), and do not consider $(x, y)$-splitters with $x=3$ or $y=3$, since otherwise we obtain a 3-int subset, and we are done.

Lemma 3 If $|I(P)|=9$ and $|V(P)|=3$, then $P$ has a 3-int subset.

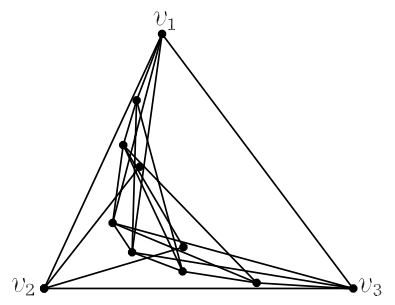

(a)

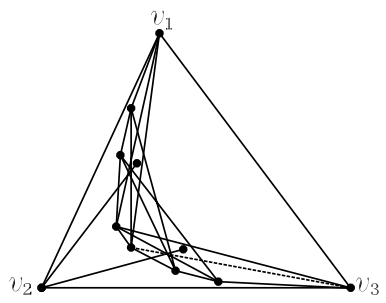

(b)

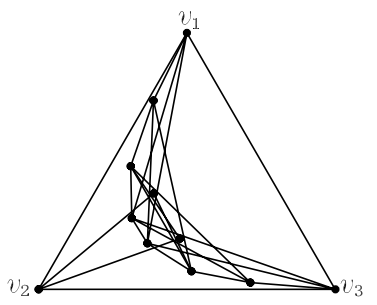

(c)

Fig. 2 (a) 8-I-monster; (b) 8-II-monster; (c) 8-III-monster 


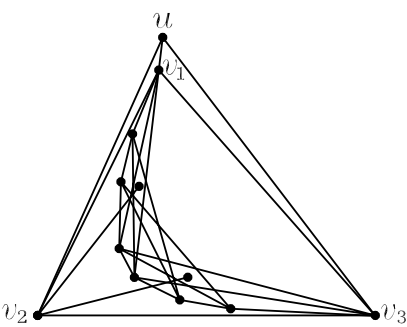

(a)

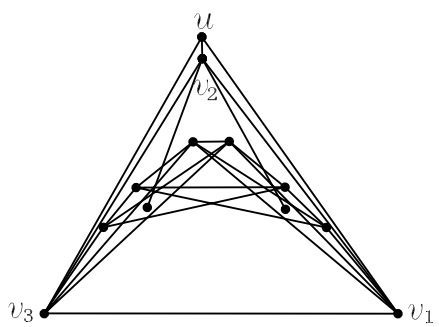

Fig. $3 \triangle v_{1} v_{2} v_{3}$ is an 8-II-monster in Fig. 2: (a) $v_{1}$ is an (8,0)-splitter; (b) $v_{2}$ is an $(8,0)$-splitter

Proof It suffices to consider $(x, y)$-splitters of $P$ of types $(8,0),(7,1),(6,2)$, and $(4,4)$.

Case $1 P$ has an $(8,0)$-splitter.

Let $\triangle v_{1} v_{2} v_{3}$ be an 8-monster as shown in Fig. 2. We may assume that $\triangle v_{1} v_{2} v_{3}$ is an 8-II-monster. The arguments for 8-I-monster and 8-III-monster are similar. Let $V\left(\triangle v_{1} v_{2} v_{3} \backslash\left\{v_{2}\right\}\right)=\left\{v_{1}, p_{1}, p_{2}, p_{3}, p_{4}, p_{5}, p_{6}, v_{3}\right\}$ and $I^{*}\left(\Delta v_{1} v_{2} v_{3} \backslash\left\{v_{2}\right\}\right)=$ $\left\{q_{1}, q_{2}\right\}$, where $q_{1} \in C\left(v_{2} ; p_{2}, p_{3}\right)$ and $q_{2} \in C\left(v_{2} ; p_{4}, p_{5}\right)$.

(a) $v_{1}$ is an $(8,0)$-splitter, $V(P)=\left\{u, v_{2}, v_{3}\right\}$, and $i^{*}\left(\triangle u v_{2} v_{3}\right)=9$. See Fig. 3a, in which $\triangle v_{1} v_{2} v_{3}$ is an 8-II-monster as shown in Fig. 2. Let $r_{u} \in I^{*}\left(\triangle u v_{2} v_{3}\right)$ be such that $C\left(u ; v_{2}, r_{u}\right) \approx \emptyset$.

If $r_{u}=v_{1}$, then $I^{*}\left(\Delta u v_{2} q_{1}\right)=\left\{v_{1}, p_{1}, p_{2}\right\}$. If $r_{u}=p_{1}$, then $I^{*}\left(\Delta u v_{2} v_{3} \backslash\right.$ $\left.\left\{v_{2}\right\}\right)=\left\{v_{1}, q_{1}, q_{2}\right\}$. If $r_{u}=p_{2}$, then $I^{*}\left(\left\{u, p_{2}, q_{2}, v_{3}\right\}\right)=\left\{v_{1}, q_{1}, p_{1}\right\}$. Next suppose that $r_{u}=p_{3}$. If $v_{1} \in H\left(q_{2} u ; v_{3}\right)$, then $I^{*}\left(\left\{u, p_{3}, q_{2}, v_{1}\right\}\right)=\left\{p_{1}, p_{2}, q_{1}\right\}$. Otherwise $I^{*}\left(\triangle u p_{2} q_{2}\right)=\left\{v_{1}, p_{1}, q_{1}\right\}$. Now assume that $r_{u}=p_{4}$. If $p_{1} \in$ $H\left(q_{2} u ; v_{3}\right)$, then $I^{*}\left(\left\{u, p_{4}, q_{2}, p_{1}\right\}\right)=\left\{p_{2}, p_{3}, q_{1}\right\}$. Otherwise, if $v_{1} \in$ $H\left(q_{2} u ; v_{3}\right)$, then $I^{*}\left(\triangle u p_{3} q_{2}\right)=\left\{p_{1}, p_{2}, q_{1}\right\} ;$ if $v_{1} \in H\left(q_{2} u ; v_{2}\right)$, then $I^{*}\left(\triangle u p_{2} q_{2}\right)=\left\{v_{1}, p_{1}, q_{1}\right\}$. Lastly consider the case where $r_{u}=p_{5}$ or $p_{6}$. If $q_{1} \in$ $H\left(p_{2} u ; v_{2}\right)$, then $I^{*}\left(\Delta u q_{1} v_{3}\right)=\left\{v_{1}, p_{1}, p_{2}\right\}$. Otherwise, if $q_{1} \in C\left(u ; p_{2}, p_{1}\right)$, then $I^{*}\left(\triangle u p_{5} q_{1}\right)=\left\{p_{2}, p_{3}, p_{4}\right\}$; if $q_{1} \in C\left(u ; p_{1}, v_{1}\right)$, then $I^{*}\left(\triangle u p_{4} q_{1}\right)=$ $\left\{p_{1}, p_{2}, p_{3}\right\}$; if $q_{1} \in H\left(u v_{1} ; v_{3}\right)$, then $I^{*}\left(\triangle u p_{3} q_{1}\right)=\left\{v_{1}, p_{1}, p_{2}\right\}$.

(b) $v_{3}$ is an $(8,0)$-splitter, $V(P)=\left\{u, v_{1}, v_{2}\right\}, i^{*}\left(\triangle u v_{1} v_{2}\right)=9 . \triangle v_{1} v_{2} v_{3}$ is an 8-IImonster as shown in Fig. 2. The proof is similar to that of (a).

(c) $v_{2}$ is a $(8,0)$-splitter, $V(P)=\left\{u, v_{3}, v_{1}\right\}$, and $i^{*}\left(\triangle u v_{3} v_{1}\right)=9$. See Fig. 3b in which $\triangle v_{1} v_{2} v_{3}$ is an 8-II-monster as shown in Fig. 2.

If $v_{2} \in H\left(p_{3} u ; v_{1}\right)$, then $I^{*}\left(\triangle u p_{3} v_{1}\right)=\left\{v_{2}, p_{1}, p_{2}\right\}$. So we can assume that $v_{2} \in H\left(p_{3} u ; v_{3}\right)$. If $v_{2} \in H\left(p_{6} u ; v_{1}\right)$, then $I^{*}\left(\triangle u p_{6} p_{3}\right)=\left\{v_{2}, p_{4}, p_{5}\right\}$. Otherwise $I^{*}\left(\triangle u v_{3} p_{4}\right)=\left\{v_{2}, p_{5}, p_{6}\right\}$.

Case $2 P$ has a $(7,1)$-splitter but no $(8,0)$-splitter.

Let $V(P)=\left\{v_{1}, v_{2}, v_{3}\right\}$. Assume that $u_{1}$ is a $(7,1)$-splitter, where $i^{*}\left(\triangle u_{1} v_{2} v_{3}\right)=$ $7, i^{*}\left(\triangle v_{1} u_{1} v_{3}\right)=1$ with $I^{*}\left(\triangle v_{1} u_{1} v_{3}\right)=\left\{u_{2}\right\}$, and $u_{2} \in H\left(v_{2} u_{1} ; v_{3}\right)$. If $u_{2}$ is a $(4,4)$-splitter of $\Delta v_{1} v_{2} v_{3}$, then $i^{*}\left(\Delta v_{2} u_{2} u_{1}\right)=3$. If $u_{2}$ is a $(6,2)$-splitter of $\triangle v_{1} v_{2} v_{3}$, then by Lemma 2, there exists a 3-int subset. For example, suppose that $i^{*}\left(\triangle v_{2} v_{3} u_{2}\right)=6$, then by Lemma 2 , the edge $v_{2} u_{2}$ is of type-2 in $\triangle v_{2} v_{3} u_{2}$, that is, there exists a subset $Q$ with $v_{2} u_{2}$ as an edge such that $i^{*}(Q)=2$, as shown in the 


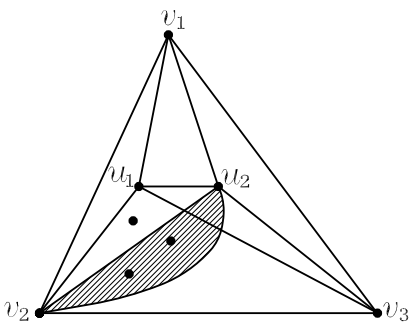

(a)

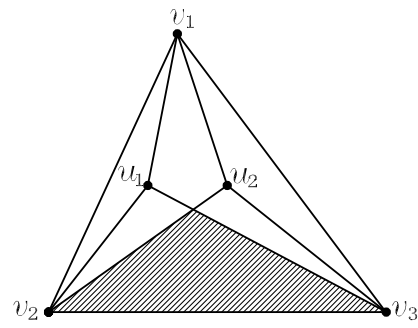

Fig. 4 (a) The convex hull of the union of the shaded region and $u_{1}$ contains 3 interior points; (b) Both $\triangle u_{1} v_{2} v_{3}$ and $\triangle u_{2} v_{2} v_{3}$ are 7 -monsters

shaded region of Fig. 4a, and $i^{*}\left(Q \cup\left\{u_{1}\right\}\right)=3$. If $u_{2}$ is a $(7,1)$-splitter of $\triangle v_{1} v_{2} v_{3}$, suppose that $i^{*}\left(\triangle v_{1} v_{2} u_{2}\right)=7$, then by Lemma 2, there exists a 3-int subset. So assume that $i^{*}\left(\triangle u_{2} v_{2} v_{3}\right)=7$. Thus both $\triangle u_{1} v_{2} v_{3}$ and $\triangle u_{2} v_{2} v_{3}$ are 7-monsters. See Fig. 4b.

Let $w_{1} \in I^{*}\left(\triangle v_{2} v_{3} u_{2}\right)$ be such that $C\left(v_{2} ; v_{3}, w_{1}\right) \approx \emptyset$, where $w_{1}$ is a $(6,0)$-, $(5,1)$ - or $(4,2)$-splitter of $\Delta u_{2} v_{2} v_{3}$, and $w_{1}$ is a $(7,1)-,(6,2)$-, or $(4,4)$-splitter of $\triangle v_{1} v_{2} v_{3}$.

Assume that $w_{1} \in H\left(v_{1} u_{1} ; v_{2}\right)$ (for $w_{1} \in C\left(v_{1} ; u_{1}, u_{2}\right)$ or $w_{1} \in H\left(v_{1} u_{2} ; v_{3}\right)$, the proof is similar).

Suppose that $w_{1}$ is a $(6,0)$-splitter of $\triangle u_{2} v_{2} v_{3}$. If $i^{*}\left(\triangle u_{2} w_{1} v_{3}\right)=6$, then $w_{1}$ is an $(8,0)$-splitter of $\triangle v_{1} v_{2} v_{3}$, contradicts to our assumption. If $i^{*}\left(\triangle u_{2} v_{2} w_{1}\right)=6$, then by Lemma 2, we can find a 3-int subset.

Suppose that $w_{1}$ is a $(5,1)$-splitter of $\triangle u_{2} v_{2} v_{3}$. If $w_{1}$ is a $(7,1)$ - or $(6,2)$ splitter of $\triangle v_{1} v_{2} v_{3}$, then $i^{*}\left(\Delta v_{1} w_{1} v_{3}\right)=7$ or 6 , and we can find a 3-int subset as before. So we only need to consider the case where $w_{1}$ is a $(4,4)$-splitter of $\triangle v_{1} v_{2} v_{3}$. Then $i^{*}\left(\triangle u_{2} v_{2} w_{1}\right)=5$ and $i^{*}\left(\triangle u_{2} w_{1} v_{3}\right)=1$, say $I^{*}\left(\triangle u_{2} w_{1} v_{3}\right)=\left\{w_{2}\right\}$. If $w_{2} \in H\left(v_{1} u_{2} ; v_{2}\right)$, then applying Lemma 2 to $\triangle u_{2} v_{2} v_{3}$, we can find a 3-int subset of $P$. So assume that $w_{2} \in H\left(v_{1} u_{2} ; v_{3}\right)$. Then $i^{*}\left(\triangle v_{1} w_{1} w_{2}\right)=3$.

Suppose that $w_{1}$ is a $(4,2)$-splitter of $\Delta u_{2} v_{2} v_{3}$. As before, if $w_{1}$ is a $(7,1)$ or $(6,2)$-splitter of $\triangle v_{1} v_{2} v_{3}$, then $i^{*}\left(\Delta v_{1} w_{1} v_{3}\right)=7$ or 6 , and we can find a 3 -int subset. So we only need to consider the case where $w_{1}$ is a $(4,4)$-splitter of $\Delta v_{1} v_{2} v_{3}$. Then $i^{*}\left(\Delta u_{2} v_{2} w_{1}\right)=4$ and $i^{*}\left(\Delta u_{2} w_{1} v_{3}\right)=2$, say $I^{*}\left(\Delta u_{2} w_{1} v_{3}\right)=$ $\left\{w_{2}, w_{3}\right\}$. We may assume that at least one of $w_{2}, w_{3}$, say $w_{2} \in H\left(v_{1} u_{2} ; v_{3}\right)$, since otherwise by applying Lemma 2 to $\triangle u_{2} v_{2} v_{3}$ we can find a 3-int subset. If $w_{3} \in$ $I^{*}\left(\triangle v_{1} w_{1} w_{2}\right)$, then $i^{*}\left(\Delta v_{1} w_{1} w_{2}\right)=3$. If $w_{3} \notin I^{*}\left(\triangle v_{1} w_{1} w_{2}\right)$, then $i^{*}\left(\triangle v_{1} w_{1} w_{2}\right)=$ 2 and $i^{*}\left(\triangle v_{1} w_{1} u_{2}\right)=1$. By property (1) of Lemma 1 , the edge $v_{1} w_{1}$ is of type-1 or type-2 in $\triangle v_{1} v_{2} w_{1}$, that is, there exists a subset $Q$ with $v_{1} w_{1}$ as an edge such that $i^{*}(Q)=1$ or 2 . Thus $i^{*}\left(Q \cup\left\{w_{2}\right\}\right)=3$ or $i^{*}\left(Q \cup\left\{u_{2}\right\}\right)=3$.

Case $3 P$ has a $(6,2)$-splitter, but no $(8,0)$-splitter and no $(7,1)$-splitter.

Let $V(P)=\left\{v_{1}, v_{2}, v_{3}\right\}$. Let $u_{1}$ be a $(6,2)$-splitter, where $i^{*}\left(\triangle u_{1} v_{2} v_{3}\right)=6$ and $i^{*}\left(\triangle v_{1} u_{1} v_{3}\right)=2$, and 2 interior points of $\triangle v_{1} u_{1} v_{3}$ lie in $H\left(v_{2} u_{1} ; v_{3}\right)$. Let $u_{2} \in I^{*}\left(\triangle v_{1} u_{1} v_{3}\right)$ such that $I^{*}\left(\triangle v_{1} u_{2} v_{3}\right)=\emptyset$, where $u_{2}$ is a $(1,0)$-splitter of $\triangle v_{1} u_{1} v_{3}$. We can assume that $i^{*}\left(\triangle v_{1} u_{1} u_{2}\right)=1$ with $I^{*}\left(\triangle v_{1} u_{1} u_{2}\right)=\{u\}$, and $u$ lies in $H\left(v_{3} u_{2} ; v_{2}\right)$, since otherwise by applying Lemma 2 to $\Delta u_{1} v_{2} v_{3}$ we obtain a 3-int 


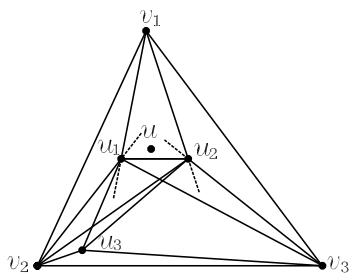

(a)

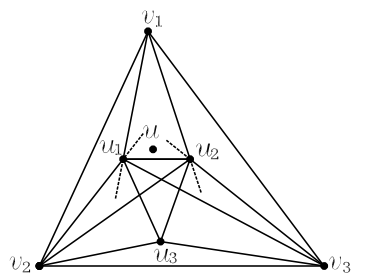

(b)

Fig. $5 i^{*}\left(\triangle u_{1} v_{2} v_{3}\right)=6$, and $I^{*}\left(\triangle u_{3} v_{2} v_{3}\right)=\emptyset$

subset. See Fig. 5. Let $u_{3} \in I^{*}\left(\triangle v_{2} v_{3} u_{2}\right)$ be such that $C\left(v_{2} ; v_{3}, u_{3}\right) \approx \emptyset$, where $u_{3}$ is a $(5,0)$-splitter or $(4,1)$-splitter of $\triangle u_{1} v_{2} v_{3}$.

(a) $u_{3} \in H\left(v_{1} u_{1} ; v_{2}\right)$ (see Fig. 5a).

Assume that $u_{3}$ is a $(4,1)$-splitter of $\triangle u_{1} v_{2} v_{3}$. If $i^{*}\left(\triangle u_{1} u_{3} v_{3}\right)=4$, then $u_{3}$ is an $(8,0)$ - or $(7,1)$-splitter of $\triangle v_{1} v_{2} v_{3}$, contradicting the assumption. So assume that $i^{*}\left(\triangle u_{1} v_{2} u_{3}\right)=4$ and $i^{*}\left(\triangle u_{1} u_{3} v_{3}\right)=1$ with $I^{*}\left(\triangle u_{1} u_{3} v_{3}\right)=\{w\}$. First suppose that $w \in H\left(u_{3} u_{2} ; u_{1}\right)$. If $u_{2}$ is a $(4,4)$-splitter of $\triangle v_{1} v_{2} v_{3}$, then $i^{*}\left(\triangle v_{2} u_{3} u_{2}\right)=3$. If $u_{2}$ is a $(6,2)$-splitter of $\triangle v_{1} v_{2} v_{3}$, then by Lemma 2, $P$ has a 3-int subset. Secondly suppose that $w \in H\left(u_{3} u_{2} ; v_{3}\right)$. If $u_{3}$ is a $(6,2)$-splitter of $\triangle v_{1} v_{2} v_{3}$, then $i^{*}\left(\triangle v_{1} u_{3} v_{3}\right)=6$, and hence $i^{*}\left(\Delta v_{1} u_{3} u\right)=3$. If $u_{3}$ is a $(4,4)$ splitter of $\triangle v_{1} v_{2} v_{3}$, then applying property (1) of Lemma 1 to $\Delta v_{1} v_{2} u_{3}$, the edge $v_{1} u_{3}$ is of type-1 (or type-2) in $\triangle v_{1} v_{2} u_{3}$, that is, there exists a subset $Q$ with $v_{1} u_{3}$ as an edge such that $i^{*}(Q)=1$ (or 2) and $i^{*}\left(Q \cup\left\{u_{2}\right\}\right)=3\left(\right.$ or $\left.i^{*}(Q \cup\{u\})=3\right)$.

Assume that $u_{3}$ is a $(5,0)$-splitter of $\triangle u_{1} v_{2} v_{3}$. Then $i^{*}\left(\triangle u_{1} v_{2} u_{3}\right)=5$, since otherwise $u_{3}$ is a $(8,0)$-splitter of $\triangle v_{1} v_{2} v_{3}$. If $u_{3}$ is a $(4,4)$-splitter of $\triangle v_{1} v_{2} v_{3}$, then $i^{*}\left(\Delta v_{1} u_{3} u_{2}\right)=3$. If $u_{3}$ is a $(6,2)$-splitter of $\triangle v_{1} v_{2} v_{3}$, then $i^{*}\left(\triangle v_{1} u_{3} v_{3}\right)=6$, and hence $i^{*}\left(\triangle u_{1} v_{1} u_{3}\right)=3$.

(b) $u_{3} \in H\left(v_{1} u_{1} ; v_{3}\right)$ (see Fig. 5b).

Assume that $u_{3}$ is a $(4,1)$-splitter of $\triangle u_{1} v_{2} v_{3}$. Then $i^{*}\left(\triangle u_{1} v_{2} u_{3}\right)=1$, say $I^{*}\left(\triangle u_{1} v_{2} u_{3}\right)=\{w\}$, since otherwise $i^{*}\left(\left\{v_{1}, u_{1}, u_{3}, v_{3}\right\}\right)=3$. If $w \in H\left(v_{1} u_{1} ; v_{3}\right)$, then applying Lemma 2 to $\triangle u_{1} v_{2} v_{3}$, we obtain a 3-int subset. So we can assume that $w \in H\left(v_{1} u_{1} ; v_{2}\right)$. If $w$ is a $(4,4)$-splitter of $\triangle v_{1} v_{2} v_{3}$, then $i^{*}\left(\triangle w u_{3} v_{3}\right)=3$. If $w$ is a $(6,2)$-splitter of $\Delta v_{1} v_{2} v_{3}$, then $i^{*}\left(\triangle v_{1} w v_{3}\right)=6$, and hence $i^{*}\left(\triangle u_{1} w v_{3}\right)=3$.

Assume that $u_{3}$ is a $(5,0)$-splitter of $\triangle u_{1} v_{2} v_{3}$. Then $i^{*}\left(\Delta_{1} v_{2} u_{3}\right)=5$, since otherwise by applying Lemma 2 to $\triangle u_{1} v_{2} v_{3}$ we obtain a 3-int subset. If $u_{2}$ is a $(4,4)$-splitter of $\triangle v_{1} v_{2} v_{3}$, then $i^{*}\left(\Delta v_{2} u_{3} u_{2}\right)=3$. If $u_{2}$ is a $(6,2)$-splitter, and if $u_{3} \in H\left(v_{1} u_{2} ; v_{2}\right)$, then due to Lemma 2, there exists a 3 -int subset; and if $u_{3} \in H\left(v_{1} u_{2} ; v_{3}\right)$, then $u_{3}$ is an $(8,0)$-splitter of $\triangle v_{1} v_{2} v_{3}$, a contradiction to our assumption.

Case 4 Every $(x, y)$-splitter is a $(4,4)$-splitter.

Let $V(P)=\left\{v_{1}, v_{2}, v_{3}\right\}$. Let $u_{1}$ be a $(4,4)$-splitter, where $i^{*}\left(\triangle v_{1} u_{1} v_{3}\right)=4$ and $i^{*}\left(\triangle u_{1} v_{2} v_{3}\right)=4$. No point lies in $H\left(v_{2} u_{1} ; v_{1}\right)$, and no point lies in $H\left(v_{1} u_{1} ; v_{2}\right)$. Let $u_{2} \in I^{*}\left(\triangle v_{1} u_{1} v_{3}\right)$ such that $I^{*}\left(\triangle v_{1} u_{2} v_{3}\right)=\emptyset$, where $u_{2}$ is a $(2,1)$-splitter of $\triangle v_{1} u_{1} v_{3}$. No point lies in $H\left(v_{3} u_{2} ; v_{1}\right)$, and no point lies in $H\left(v_{1} u_{2} ; v_{3}\right)$. Let 


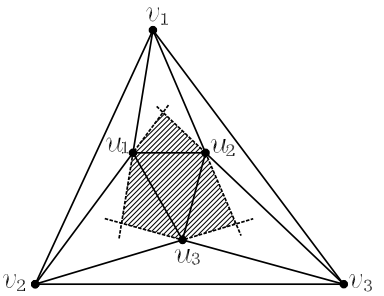

(a)

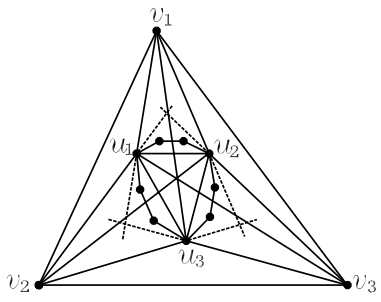

(b)

Fig. 6 (a) The points of $I(P)$ lie in the shaded region; (b) $I(P)$ is a convex 9-gon

$u_{3} \in I^{*}\left(\triangle u_{1} v_{2} v_{3}\right)$ be such that $I^{*}\left(\triangle u_{3} v_{2} v_{3}\right)=\emptyset$, where $u_{3}$ is a $(2,1)$-splitter of $\triangle u_{1} v_{2} v_{3}$. No point lies in $H\left(v_{3} u_{3} ; v_{2}\right)$, and no point lies in $H\left(v_{2} u_{3} ; v_{3}\right)$. See Fig. 6a. So we may assume that either $i^{*}\left(\triangle u_{1} u_{3} v_{3}\right)=i^{*}\left(\triangle u_{1} v_{3} u_{2}\right)=1$ or $i^{*}\left(\triangle u_{1} u_{3} v_{3}\right)=$ $i^{*}\left(\triangle u_{1} v_{3} u_{2}\right)=2$, since otherwise $i^{*}\left(\left\{u_{1}, u_{3}, v_{3}, u_{2}\right\}\right)=3$.

(a) $i^{*}\left(\triangle u_{1} u_{3} v_{3}\right)=i^{*}\left(\triangle u_{1} v_{3} u_{2}\right)=2$.

Then $i^{*}\left(\triangle v_{1} u_{1} u_{2}\right)=i^{*}\left(\triangle v_{2} u_{3} u_{1}\right)=1$. If $i^{*}\left(\triangle u_{1} u_{3} u_{2}\right)=4$, then $i^{*}\left(\left\{v_{1}, u_{1}\right.\right.$, $\left.\left.w, u_{2}\right\}\right)=3$ or $i^{*}\left(\left\{v_{2}, u_{3}, w, u_{1}\right\}\right)=3$, where $w \in I^{*}\left(\triangle u_{1} u_{3} u_{2}\right)$ such that $I^{*}\left(\triangle w u_{3} u_{2}\right)=\emptyset$, and $w$ is a $(2,1)$-splitter of $\triangle u_{1} u_{3} u_{2}$. If $i^{*}\left(\triangle u_{1} u_{3} u_{2}\right)=3$, then we are done. If $i^{*}\left(\triangle u_{1} u_{3} u_{2}\right)=2$, then $i^{*}\left(\left\{v_{1}, u_{1}, u_{3}, u_{2}\right\}\right)=3$. If $i^{*}\left(\triangle u_{1} u_{3} u_{2}\right)=1$, then $i^{*}\left(\triangle u_{3} v_{3} u_{2}\right)=3$. If $i^{*}\left(\triangle u_{1} u_{3} u_{2}\right)=0$, then $i^{*}\left(\left\{v_{1}, v_{2}, u_{3}, u_{2}\right\}\right)=3$.

(b) $i^{*}\left(\triangle u_{1} u_{3} v_{3}\right)=i^{*}\left(\triangle u_{1} v_{3} u_{2}\right)=1$.

Then $i^{*}\left(\triangle v_{1} u_{1} u_{2}\right)=i^{*}\left(\triangle v_{2} u_{3} u_{1}\right)=2$. If $i^{*}\left(\triangle u_{1} u_{3} u_{2}\right)=2$, then $i^{*}\left(\left\{v_{1}, u_{1}\right.\right.$, $\left.\left.w, u_{2}\right\}\right)=3$ or $i^{*}\left(\left\{v_{2}, u_{3}, w, u_{1}\right\}\right)=3$, where $w \in I^{*}\left(\triangle u_{1} u_{3} u_{2}\right)$ such that $I^{*}\left(\triangle w u_{3} u_{2}\right)=\emptyset$, and $w$ is a $(1,0)$-splitter of $\triangle u_{1} u_{3} u_{2}$. If $i^{*}\left(\triangle u_{1} u_{3} u_{2}\right)=1$, then $i^{*}\left(\left\{v_{1}, u_{1}, u_{3}, u_{2}\right\}\right)=3$. If $i^{*}\left(\triangle u_{1} u_{3} u_{2}\right)=0$, then $I(P)$ is a convex 9-gon, see Fig. 6b, since otherwise there exists a 3 -int subset. Then it is easy to see that $P$ has a 3 -int subset.

Lemma 4 If $|I(P)|=9$ and $|V(P)| \geq 4$, then $P$ has a 3-int subset.

Proof Let $V(P)=\left\{v_{1}, v_{2}, \ldots, v_{m}\right\}$, where $m=|V(P)|$. Triangulate $P$ by joining vertex $v_{1}$ to each of the other vertices in $V(P)$, and we obtain $m-2$ triangles. If there exists a triangle $\triangle$ such that $i^{*}(\triangle)=3$ or 9 , we are done. If there exists a triangle $\triangle$ such that $i^{*}(\triangle)=6,7$, or 8 , due to Lemma 2, there exists a 3-int subset. If for any triangle $\triangle, i^{*}(\triangle) \leq 4$, then due to Lemma 1 it is easy to verify that we may find a 3-int subset of $P$ by concatenating a set of adjacent triangles. If one triangle contains 5 interior points and each of the other triangles contains at most 2 interior points, then by Lemma 1 , it is easy to verify that we may find a 3-int subset. So we can assume that $i^{*}\left(\triangle v_{1} v_{i} v_{i+1}\right)=5$ and $i^{*}\left(\triangle v_{1} v_{j} v_{j+1}\right)=4$, and the other remaining triangles contain no interior point, where $i+1 \leq j$.

Case 1 The two triangles $\triangle v_{1} v_{i} v_{i+1}$ and $\Delta v_{1} v_{j} v_{j+1}$ are adjacent.

Without loss of generality, we may assume that $i^{*}\left(\triangle v_{1} v_{2} v_{3}\right)=5$ and $i^{*}\left(\triangle v_{1} v_{3} v_{4}\right)=4$. By Lemma 1 , it suffices to consider the case where $\triangle v_{1} v_{2} v_{3}$ is a 5-I-monster, that is, the edge $v_{1} v_{3}$ is of type- 2 but not of type- 1 in $\triangle v_{1} v_{2} v_{3}$, or 

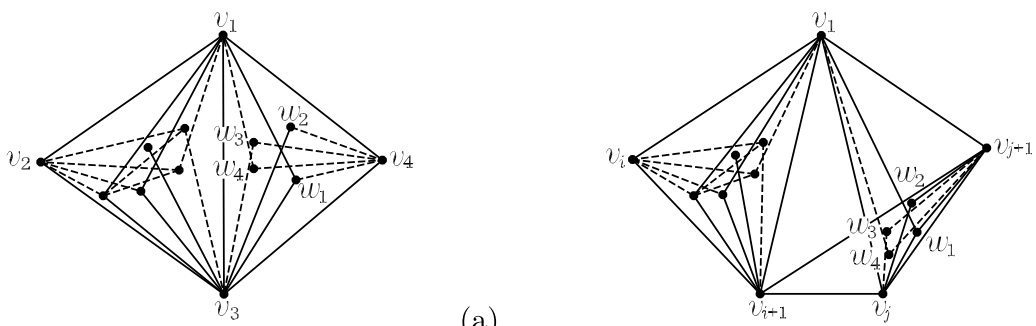

Fig. $7 \triangle v_{1} v_{2} v_{3}$ is a 5-I-monster

a 5-II-monster, that is, the edge $v_{1} v_{3}$ is of type- 1 but not of type- 2 in $\triangle v_{1} v_{2} v_{3}$. If $i^{*}\left(\triangle v_{1} v_{2} v_{4}\right) \geq 6$ or $\leq 3$, then by Lemma 3 or Lemma $2, P$ has a 3 -int subset. So assume that $i^{*}\left(\triangle v_{1} v_{2} v_{4}\right)=4$ or 5 .

(a) $\triangle v_{1} v_{2} v_{3}$ is a 5-I-monster. See Fig. 1a.

Rotate the edge $v_{1} v_{3}$ counter clockwise with $v_{3}$ as center in $\Delta v_{1} v_{2} v_{3}$, and label the points the edge $v_{1} v_{3}$ meets consecutively by $a_{1}, a_{2}, a_{3}, a_{4}, a_{5}$.

Let $w_{1} \in I^{*}\left(\triangle v_{1} v_{3} v_{4}\right)$ such that $C\left(v_{3} ; v_{4}, w_{1}\right) \approx \emptyset$, where $w_{1}$ is a $(2,1)$ splitter of $\triangle v_{1} v_{3} v_{4}$. If $i^{*}\left(\Delta v_{1} v_{3} w_{1}\right)=1$, then $i^{*}\left(\left\{v_{1}, a_{3}, v_{3}, w_{1}\right\}\right)=3$. So assume that $i^{*}\left(\triangle v_{1} w_{1} v_{4}\right)=1$ with $I^{*}\left(\triangle v_{1} w_{1} v_{4}\right)=\left\{w_{2}\right\}$ and $i^{*}\left(\triangle v_{1} v_{3} w_{1}\right)=2$ with $I^{*}\left(\triangle v_{1} v_{3} w_{1}\right)=\left\{w_{3}, w_{4}\right\}$. Then $\left\{v_{1}, v_{3}, w_{4}, w_{3}\right\}$ is convex, since otherwise $I^{*}\left(\left\{v_{1}, a_{3}, v_{3}, w_{3}\right\}\right)=\left\{a_{1}, a_{2}, w_{4}\right\}$ or $I^{*}\left(\left\{v_{1}, a_{3}, v_{3}, w_{4}\right\}\right)=\left\{a_{1}, a_{2}, w_{3}\right\}$. Suppose that $w_{3}, w_{4} \in H\left(v_{3} w_{2} ; v_{1}\right)$, since otherwise $I^{*}\left(\left\{v_{1}, a_{3}, v_{3}, w_{2}\right\}\right)=$ $\left\{a_{1}, a_{2}, w_{3}\right\}$ or $I^{*}\left(\triangle v_{3} v_{4} w_{2}\right)=\left\{w_{1}, w_{3}, w_{4}\right\}$. Then $w_{3}, w_{4} \in C\left(v_{4} ; w_{1}, w_{2}\right)$, since otherwise $I^{*}\left(\Delta v_{3} v_{4} w_{3}\right)=\left\{w_{1}, w_{2}, w_{4}\right\}$ or $I^{*}\left(\triangle v_{1} w_{4} v_{4}\right)=\left\{w_{1}, w_{2}, w_{3}\right\}$. See Fig. 7a.

Suppose that $a_{3} \in H\left(v_{2} v_{4} ; v_{1}\right)$ and $w_{4} \in H\left(v_{2} v_{4} ; v_{3}\right)$. If $a_{2} \in H\left(a_{3} w_{4} ; v_{3}\right)$, then $I^{*}\left(\left\{v_{2}, v_{3}, w_{4}, a_{3}\right\}\right)=\left\{a_{2}, a_{4}, a_{5}\right\}$. Otherwise $I^{*}\left(\left\{v_{1}, a_{3}, w_{4}, w_{2}\right\}\right)=$ $\left\{a_{1}, a_{2}, w_{3}\right\}$. Suppose that $a_{3} \in H\left(v_{2} v_{4} ; v_{3}\right)$ and $w_{4} \in H\left(v_{2} v_{4} ; v_{1}\right)$. If $w_{3}, w_{4} \in$ $H\left(a_{2} w_{2} ; v_{1}\right)$, then $I^{*}\left(\left\{v_{1}, a_{3}, a_{2}, w_{2}\right\}\right)=\left\{a_{1}, w_{3}, w_{4}\right\}$; if $w_{3}, w_{4} \in H\left(a_{2} w_{2} ; v_{3}\right)$, then $I^{*}\left(\left\{v_{3}, v_{4}, w_{2}, a_{2}\right\}\right)=\left\{w_{1}, w_{3}, w_{4}\right\} ;$ otherwise $I^{*}\left(\left\{v_{1}, v_{2}, a_{2}, w_{2}\right\}\right)=$ $\left\{a_{1}, a_{3}, w_{3}\right\}$. Suppose that $a_{3}, w_{4} \in H\left(v_{2} v_{4} ; v_{1}\right)$. Then $I^{*}\left(\triangle v_{1} v_{2} v_{4}\right)=\left\{a_{1}, a_{3}\right.$, $\left.w_{2}, w_{3}, w_{4}\right\}$. If $w_{3}, w_{4} \in H\left(a_{1} w_{1} ; v_{1}\right)$, then $I^{*}\left(\left\{v_{1}, a_{1}, w_{1}, v_{4}\right\}\right)=\left\{w_{2}, w_{3}, w_{4}\right\}$; if $w_{3}, w_{4} \in H\left(a_{1} w_{1} ; v_{3}\right)$, then $I^{*}\left(\left\{a_{1}, a_{3}, v_{3}, w_{1}\right\}\right)=\left\{a_{2}, w_{3}, w_{4}\right\}$; otherwise $I^{*}\left(\left\{a_{1}, a_{5}, v_{3}, w_{1}\right\}\right)=\left\{a_{2}, a_{4}, w_{4}\right\}$. Suppose that $a_{3}, w_{4} \in H\left(v_{2} v_{4} ; v_{3}\right)$. Then $i^{*}\left(\triangle v_{2} v_{3} v_{4}\right) \geq 6$, and we are done.

(b) $\triangle v_{1} v_{2} v_{3}$ is a 5-II-monster (see Fig. 1b).

Rotate the edge $v_{1} v_{3}$ counter clockwise with $v_{3}$ as center in $\Delta v_{1} v_{2} v_{3}$, and label the points the edge $v_{1} v_{3}$ meets consecutively by $a_{1}, a_{2}, a_{3}, a_{4}, a_{5}$.

Let $w_{1} \in I^{*}\left(\triangle v_{1} v_{3} v_{4}\right)$ be such that $C\left(v_{3} ; v_{4}, w_{1}\right) \approx \emptyset$, where $w_{1}$ is a $(2,1)$ splitter of $\triangle v_{1} v_{3} v_{4}$. Then assume that $i^{*}\left(\triangle v_{1} v_{3} w_{1}\right)=1$ with $I^{*}\left(\triangle v_{1} v_{3} w_{1}\right)=$ $\left\{w_{2}\right\}$ and $i^{*}\left(\triangle v_{1} w_{1} v_{4}\right)=2$ with $I^{*}\left(\triangle v_{1} w_{1} v_{4}\right)=\left\{w_{3}, w_{4}\right\}$, since otherwise $i^{*}\left(\left\{v_{1}, a_{1}, a_{5}, v_{3}, w_{1}\right\}\right)=3$. Suppose that $\left|V\left(\triangle v_{1} v_{3} v_{4} \backslash\left\{v_{4}\right\}\right)\right|=5$ with $V\left(\triangle v_{1} v_{3} v_{4} \backslash\left\{v_{4}\right\}\right)=\left\{v_{1}, v_{3}, w_{1}, w_{4}, w_{3}\right\}$, since otherwise there exists a 3 -int subset. Suppose that $w_{2} \in H\left(v_{3} w_{3} ; v_{1}\right)$, since otherwise $I^{*}\left(\triangle v_{3} v_{4} w_{3}\right)=$ $\left\{w_{1}, w_{2}, w_{4}\right\}$. See Fig. 8a. 

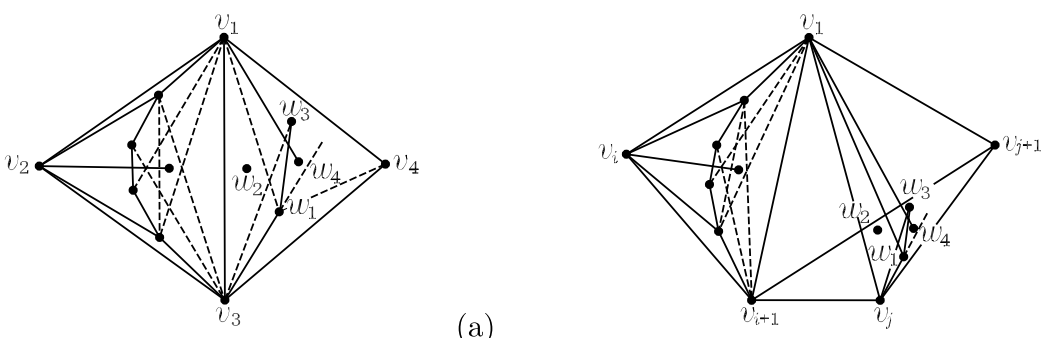

Fig. $8 \Delta v_{1} v_{2} v_{3}$ is a 5-II-monster

When $a_{2}, w_{4} \in H\left(v_{2} v_{4} ; v_{1}\right), I^{*}\left(\left\{a_{2}, v_{2}, v_{3}, w_{1}\right\}\right)=\left\{a_{4}, a_{5}, w_{2}\right\}$ or $I^{*}\left(\left\{v_{1}, a_{2}\right.\right.$, $\left.\left.w_{1}, v_{4}\right\}\right)=\left\{w_{2}, w_{3}, w_{4}\right\}$. When $a_{2}, w_{4} \in H\left(v_{2} v_{4} ; v_{3}\right), I^{*}\left(\left\{v_{1}, v_{2}, a_{2}, w_{3}\right\}\right)=$ $\left\{a_{1}, a_{3}, w_{2}\right\}$ or $I^{*}\left(\left\{a_{2}, v_{3}, v_{4}, w_{3}\right\}\right)=\left\{w_{1}, w_{2}, w_{4}\right\}$. When $a_{2} \in H\left(v_{2} v_{4} ; v_{3}\right)$ and $w_{4} \in H\left(v_{2} v_{4} ; v_{1}\right), I^{*}\left(\left\{v_{1}, v_{2}, a_{2}, w_{3}\right\}\right)=\left\{a_{1}, a_{3}, w_{2}\right\}$ or $I^{*}\left(\left\{a_{2}, v_{3}, v_{4}, w_{3}\right\}\right)=$ $\left\{w_{1}, w_{2}, w_{4}\right\}$. When $a_{2} \in H\left(v_{2} v_{4} ; v_{1}\right)$ and $w_{4} \in H\left(v_{2} v_{4} ; v_{3}\right), I^{*}\left(\left\{v_{1}, a_{2}, w_{1}, v_{4}\right\}\right)$ $=\left\{w_{2}, w_{3}, w_{4}\right\}$ or $I^{*}\left(\left\{a_{2}, v_{2}, v_{3}, w_{1}\right\}\right)=\left\{w_{2}, a_{4}, a_{5}\right\}$.

Case 2 The two triangles $\Delta v_{1} v_{i} v_{i+1}$ and $\Delta v_{1} v_{j} v_{j+1}$ are not adjacent.

Due to Lemma 1, it suffices to consider the case where $\Delta v_{1} v_{i} v_{i+1}$ is a 5-I-monster or a 5-II-monster. Connect $v_{i+1}$ and $v_{j+1}$. If $i^{*}\left(\triangle v_{1} v_{i+1} v_{j+1}\right) \geq 1$, then the conclusion is correct as before. So suppose that $i^{*}\left(\Delta v_{i+1} v_{j} v_{j+1}\right)=4$. Rotate the edge $v_{1} v_{i+1}$ counter clockwise with $v_{i+1}$ as center in $\Delta v_{1} v_{i} v_{i+1}$, and label the points the edge $v_{1} v_{i+1}$ meets consecutively by $a_{1}, a_{2}, a_{3}, a_{4}, a_{5}$.

(a) $\triangle v_{1} v_{i} v_{i+1}$ is a 5-I-monster.

The proof is similar to that of Case 1(a), and we label the four interior points of $\triangle v_{1} v_{j} v_{j+1}$ as shown in Fig. 7b. Then $I^{*}\left(\left\{v_{1}, a_{3}, v_{i+1}, w_{3}, v_{j+1}\right\}\right)=$ $\left\{a_{1}, a_{2}, w_{2}\right\}$.

(b) $\triangle v_{1} v_{i} v_{i+1}$ is a 5-II-monster.

The proof is similar to that of Case 1(b), and we label the four interior points of $\triangle v_{1} v_{j} v_{j+1}$ as shown in Fig. 8b. If $w_{2} \in H\left(w_{1} v_{i+1} ; v_{1}\right)$, then $I^{*}\left(\triangle v_{i+1} w_{1} v_{j+1}\right)=\left\{w_{2}, w_{3}, w_{4}\right\}$. Otherwise $I^{*}\left(\left\{v_{1}, a_{1}, a_{5}, v_{i+1}, w_{1}, v_{j+1}\right\}\right)=$ $\left\{a_{2}, w_{3}, w_{4}\right\}$.

Lemma 5 If $|I(P)|=10$ and $|V(P)|=3$, then $P$ has a 3-int subset.

Proof Let $V(P)=\left\{v_{1}, v_{2}, v_{3}\right\}$. If $P$ has a $(9,0)$-splitter, then by Lemma 3, we are done. So it remains to consider $(x, y)$-splitters of $P$ of types $(8,1)$, or $(7,2)$, or $(5,4)$.

Case $1 P$ has an $(8,1)$-splitter.

The proof is similar to that of Case 1 of Lemma 3.

Case $2 P$ has a $(7,2)$-splitter but no $(8,1)$-splitter.

Let $u_{1}$ be a $(7,2)$-splitter, where $i^{*}\left(\triangle u_{1} v_{2} v_{3}\right)=7$, and $i^{*}\left(\triangle v_{1} u_{1} v_{3}\right)=2$ with $\left.\triangle v_{1} u_{1} v_{3}\right)=\left\{u_{2}, u_{3}\right\}, u_{2}, u_{3} \in H\left(v_{2} u_{1} ; v_{3}\right)$. Let $u_{2} \in I^{*}\left(\triangle v_{1} u_{1} v_{3}\right)$ be such that $I^{*}\left(\triangle v_{1} u_{2} v_{3}\right)=\emptyset$, where $u_{2}$ is a $(1,0)$-splitter of $\triangle v_{1} u_{1} v_{3}$. Assume that $i^{*}\left(\triangle v_{1} u_{1} u_{2}\right)=1$ with $I^{*}\left(\triangle v_{1} u_{1} u_{2}\right)=\left\{u_{3}\right\}$, and $u_{3}$ lies in $H\left(v_{3} u_{2} ; u_{1}\right)$, since otherwise by applying Lemma 2 to $\triangle u_{1} v_{2} v_{3}$ we obtain a 3-int subset. 


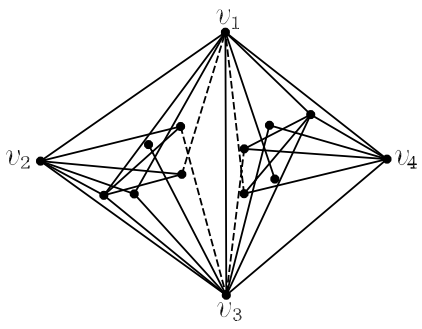

(a)

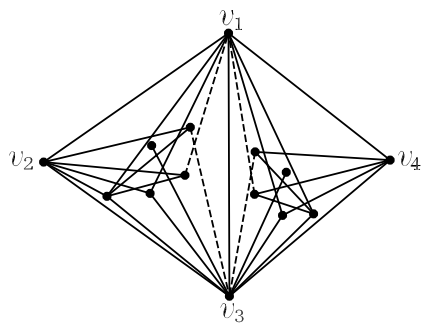

Fig. 9 Two triangles are adjacent, both $\Delta v_{1} v_{2} v_{3}$ and $\Delta v_{1} v_{3} v_{4}$ are 5-I-monsters

(a) $u_{2}$ is a $(5,4)$-splitter of $\Delta v_{1} v_{2} v_{3}$.

The proof is similar to that in the previous discussion and is omitted.

(b) $u_{2}$ is a $(7,2)$-splitter of $\triangle v_{1} v_{2} v_{3}$.

If $i^{*}\left(\triangle v_{1} v_{2} u_{2}\right)=7$, then applying Lemma 2 to $\triangle v_{1} v_{2} u_{2}$ we obtain a 3-int subset. So we may assume that $i^{*}\left(\Delta v_{2} v_{3} u_{2}\right)=7$. Thus both $\Delta u_{1} v_{2} v_{3}$ and $\Delta u_{2} v_{2} v_{3}$ are 7monsters. As in Case 2 of Lemma 3, it is easy to prove that $P$ has a 3-int subset.

Case 3 Every $(x, y)$-splitter is a $(5,4)$-splitter.

Let $u_{1}$ be a $(5,4)$-splitter, where $i^{*}\left(\Delta u_{1} v_{2} v_{3}\right)=5, i^{*}\left(\triangle v_{1} u_{1} v_{3}\right)=4$, and 4 interior points of $\triangle v_{1} u_{1} v_{3}$ lie in $H\left(v_{2} u_{1} ; v_{3}\right)$. By Lemma 1, it suffices to consider the case where $\Delta u_{1} v_{2} v_{3}$ is a 5-I-monster or a 5-II-monster. As in Case 1 of Lemma 4, we can obtain a 3 -int subset.

Lemma 6 If $|I(P)|=10$ and $|V(P)| \geq 4$, then $P$ has a 3-int subset.

Proof Let $V(P)=\left\{v_{1}, v_{2}, \ldots, v_{m}\right\}$, where $m=|V(P)|$. Similarly to Lemma 4, we need only to consider the case where there are two triangles, each of which contains 5 interior points.

Case 1 The two triangles are adjacent.

Without loss of generality, we assume that $i^{*}\left(\triangle v_{1} v_{2} v_{3}\right)=i^{*}\left(\triangle v_{1} v_{3} v_{4}\right)=5$. Due to Lemma 1 , it suffices to consider the cases where both $\triangle v_{1} v_{2} v_{3}$ and $\triangle v_{1} v_{3} v_{4}$ are 5 I-monsters or 5-II-monsters. If $i^{*}\left(\triangle v_{1} v_{2} v_{4}\right) \geq 6$ or $\leq 4$, then by Lemmas 5, 3, or 2, $P$ has a 3-int subset. So assume that $i^{*}\left(\triangle v_{1} v_{2} v_{4}\right)=5$, and hence $i^{*}\left(\triangle v_{2} v_{3} v_{4}\right)=5$ as well.

(a) Both $\triangle v_{1} v_{2} v_{3}$ and $\triangle v_{1} v_{3} v_{4}$ are 5-I-monsters.

Then the edge $v_{1} v_{3}$ is of type-2 but not of type-1 in $\Delta v_{1} v_{2} v_{3}$, and the edge $v_{1} v_{3}$ is of type-2 but not of type-1 in $\Delta v_{1} v_{3} v_{4}$. We obtain two configurations as shown in Fig. 9.

In Fig. 9a, for $\Delta v_{1} v_{2} v_{3}$, we rotate the edge $v_{1} v_{3}$ counter clockwise with $v_{3}$ as center and label the points the edge $v_{1} v_{3}$ meets consecutively by $a_{1}, a_{2}, a_{3}, a_{4}, a_{5}$; and for $\Delta v_{1} v_{3} v_{4}$, rotate the edge $v_{1} v_{4}$ counter clockwise with $v_{4}$ as center and label the points the edge $v_{1} v_{4}$ meets consecutively by $a_{6}, a_{7}, a_{8}, a_{9}, a_{0}$.

Suppose that $a_{1}, a_{9} \in H\left(v_{2} v_{4} ; v_{3}\right)$. Then $i^{*}\left(\triangle v_{2} v_{3} v_{4}\right) \geq 7$, and we are done. Suppose that $a_{1}, a_{9} \in H\left(v_{2} v_{4} ; v_{1}\right)$. Then $I^{*}\left(\triangle v_{1} v_{2} v_{4}\right)=\left\{a_{1}, a_{6}, a_{7}, a_{8}, a_{9}\right\}$. If $a_{0} \in H\left(a_{2} a_{9} ; v_{3}\right)$, then $I^{*}\left(\left\{a_{2}, a_{9}, v_{1}, v_{2}\right\}\right)=\left\{a_{1}, a_{3}, a_{8}\right\}$. Otherwise 


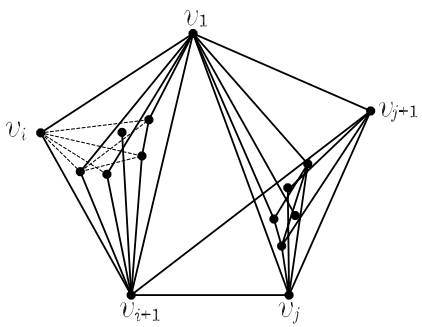

(a)

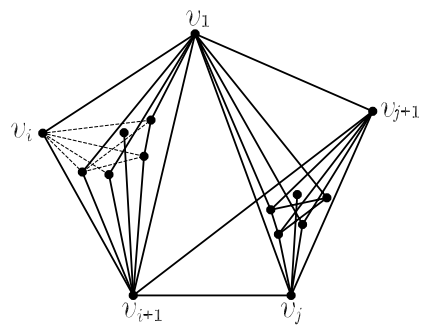

Fig. 10 Two triangles are not adjacent, both $\Delta v_{1} v_{2} v_{3}$ and $\Delta v_{1} v_{3} v_{4}$ are 5-I-monsters

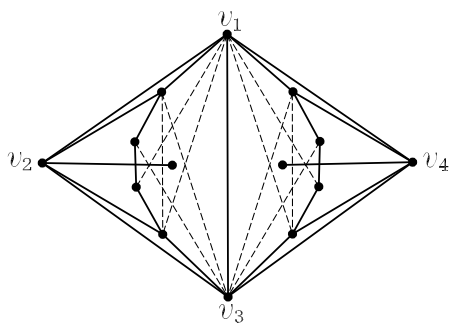

(a)

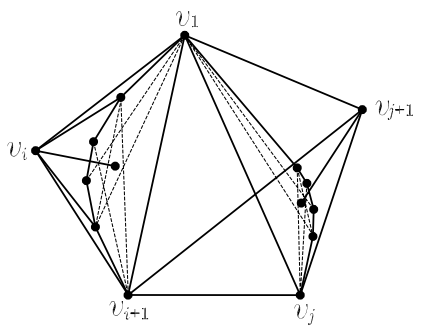

Fig. 11 Both $\Delta v_{1} v_{2} v_{3}$ and $\Delta v_{1} v_{3} v_{4}$ are 5-II-monsters

$I^{*}\left(\left\{v_{1}, a_{3}, a_{2}, a_{9}\right\}\right)=\left\{a_{1}, a_{0}, a_{8}\right\}$. Next, suppose that $a_{1} \in H\left(v_{2} v_{4} ; v_{1}\right)$ and $a_{9} \in$ $H\left(v_{2} v_{4} ; v_{3}\right)$. If $a_{8} \in H\left(a_{1} a_{9} ; v_{1}\right)$, then $I^{*}\left(\left\{v_{1}, a_{1}, a_{9}, v_{4}\right\}\right)=\left\{a_{6}, a_{7}, a_{8}\right\}$. Otherwise $I^{*}\left(\left\{a_{1}, a_{4}, v_{3}, a_{9}\right\}\right)=\left\{a_{2}, a_{0}, a_{8}\right\}$. Lastly suppose that $a_{1} \in H\left(v_{2} v_{4} ; v_{3}\right)$ and $a_{9} \in H\left(v_{2} v_{4} ; v_{1}\right)$. Then $I^{*}\left(\triangle v_{1} v_{2} v_{4}\right)=\left\{a_{6}, a_{7}, a_{8}, a_{9}, a_{0}\right\}$. If $a_{1} \in H\left(a_{3} a_{8} ; v_{3}\right)$, then $I^{*}\left(\left\{a_{3}, v_{3}, a_{9}, a_{8}\right\}\right)=\left\{a_{1}, a_{2}, a_{0}\right\}$. Otherwise $I^{*}\left(\left\{a_{3}, v_{3}, v_{4}, a_{8}\right\}\right)=$ $\left\{a_{2}, a_{0}, a_{9}\right\}$.

The proof for the case of Fig. 9b is similar to that of Fig. 9a, and we can obtain a subset $Q \subset P$ such that $i^{*}(Q)=3$.

(b) Both $\triangle v_{1} v_{2} v_{3}$ and $\triangle v_{1} v_{3} v_{4}$ are 5-II-monsters.

Then the edge $v_{1} v_{3}$ is of type- 1 but not of type- 2 in $\Delta v_{1} v_{2} v_{3}$, and the edge $v_{1} v_{3}$ is of type- 1 but not of type-2 in $\triangle v_{1} v_{3} v_{4}$ too. See Fig. 11a. By an argument similar to that of Fig. 9a, we can find a subset $Q \subset P$ such that $i^{*}(Q)=3$.

Case 2 The two triangles are not adjacent.

Without loss of generality, we assume that $i^{*}\left(\triangle v_{1} v_{i} v_{i+1}\right)=5$ and $i^{*}\left(\triangle v_{1} v_{j} v_{j+1}\right)=5$, where $i+1<j$. Due to Lemma 1 , it suffices to consider the cases where both $\triangle v_{1} v_{i} v_{i+1}$ and $\triangle v_{1} v_{j} v_{j+1}$ are 5-I-monsters (or 5-II-monsters), as in Case 1. Connect $v_{i+1}$ and $v_{j+1}$. If $i^{*}\left(\triangle v_{1} v_{i+1} v_{j+1}\right) \geq 1$, the conclusion is correct as before, and we are done. So assume that $i^{*}\left(\triangle v_{i+1} v_{j} v_{j+1}\right)=5$.

(a) Both $\triangle v_{1} v_{i} v_{i+1}$ and $\Delta v_{1} v_{j} v_{j+1}$ are 5-I-monsters.

Then, as in Case 1 (a), we obtain two configurations as shown in Fig. 10. It is easy to find 3-int subsets.

(b) Both $\triangle v_{1} v_{i} v_{i+1}$ and $\Delta v_{1} v_{j} v_{j+1}$ are 5-II-monster.

Then as shown in Fig. 11b, it is clear that $P$ has a 3 -int subset. 
Lemma 7 If $|I(P)|=11$ and $|V(P)|=3$, then $P$ has a 3-int subset.

Proof Let $V(P)=\left\{v_{1}, v_{2}, v_{3}\right\}$. If $P$ has a $(10,0)$ - or $(9,1)$-splitter, then by Lemma 5 or Lemma 3, we are done. So we need only to consider $(x, y)$-splitters of $P$ of types $(8,2)$ or $(6,4)$, or $(5,5)$. In each case, we obtain a 3 -int subset. The proof is simple on the basis of the previous discussion and is omitted.

Lemma 8 If $|I(P)|=11$ and $|V(P)| \geq 4$, then $P$ has a 3-int subset.

Proof Let $|V(P)|=m$ and $|I(P)|=s$. If $m=4$ or 5, it is easy to prove that $P$ has a 3-int subset as before. If $m \geq 6$, then the Reduction Lemma implies the existence of a proper subset $P^{\prime} \subset P$ with $9 \leq i^{*}\left(P^{\prime}\right)<|I(P)|$ : if (a) of the Reduction Lemma holds, we have a proper subset $P^{\prime} \subset P$ with $i^{*}\left(P^{\prime}\right)=10$; and if (b) of the Reduction Lemma holds, we have a proper subset $P^{\prime} \subset P$ with $i^{*}\left(P^{\prime}\right)=11-\left\lfloor\frac{11}{m}\right\rfloor-1 \geq 9$. Then by using the previous results we obtain a subset $Q \subset P^{\prime}$ with $i^{*}(Q)=3$, and hence $P$ has a 3-int subset.

Lemma 9 If $|I(P)| \geq 12$ and $|V(P)| \geq 3$, then $P$ has a 3-int subset.

Proof Let $|V(P)|=m$ and $|I(P)|=s$. Consider the case $s=12$. If $m=3$ or 4 , due to Lemmas 1 and 2, it is easy to prove the conclusion. For $m \geq 5$, we may apply the Reduction Lemma and an argument similar to that in Lemma 8. In the case $s=13$, the proof is similar to that of the case $s=12$. Finally, if $m \geq 3$ and $s \geq 14$, we have $s-\left\lfloor\frac{s}{m}\right\rfloor-1 \geq 9$, and so the result follows by using the Reduction Lemma and the similar argument as above.

Combining Lemmas 3-9, we finish the proof for $g(3) \leq 9$ and reach the conclusion that $g(3)=9$ at last.

\section{References}

1. Avis, D., Hosono, K., Urabe, M.: On the existence of a point subset with 4 or 5 interior points. In: JCDCG 1998. LNCS, vol. 1763, pp. 57-64 (2000)

2. Avis, D., Hosono, K., Urabe, M.: On the existence of a point subset with a specified number of interior points. Discrete Math. 241, 33-40 (2001)

3. Bisztriczky, T., Hosono, K., Károlyi, G., Urabe, M.: Constructions from empty polygons. Period. Math. Hung. 49, 1-8 (2004)

4. Bonnice, W.E.: On convex polygons determined by a finite planar set. Am. Math. Mon. 81, 749-752 (1974)

5. Brass, P., Moser, W., Pach, J.: Research Problems in Discrete Geometry. Springer, Berlin (2005)

6. Erdős, P., Szekeres, G.: A combinatorial problem in Geometry. Compos. Math. 2, 463-470 (1935)

7. Erdős, P., Szekeres, G.: On some extremum problems in elementary geometry. Ann. Univ. Sci. Bp. 3-4, 53-62 (1960-1961)

8. Fevens, T.: A note on point subset with a specified number of interior points. In: JCDCG 2002. LNCS, vol. 2866, pp. 152-158 (2003)

9. Hosono, K.: On the existence of a convex point subset containing one triangle in the plane. Discrete Math. 305, 201-218 (2005)

10. Hosono, K., Károlyi, G., Urabe, M.: On the existence of a convex polygon with a specified number of interior points. In: Discrete Geometry, In Honor of W. Kuperberg's 60th Birthday, pp. 351-358. Dekker, New York (2003) 
11. Kalbfleisch, J.D., Kalbfleisch, J.G., Santon, R.G.: A combinatorial problem on convex $n$-gons. In: Proc. Louisiana Conf. Comb. Graph Theory and Computing, pp. 180-188. Baton Rouge (1970)

12. Károlyi, G., Pach, J., Tóth, G.: A modular version of the Erdős-Szekeres theorem. Stud. Sci. Math. Hung. 38, 245-259 (2001)

13. Morris, W., Soltan, V.: The Erdős-Szekeres problem on point in convex position-a survey. Bull. New Ser. Am. Math. Soc. 37, 437-458 (2000)

14. Pach, J., Tóth, G.: A generalization of the Erdős-Szekeres theorem to disjoint convex sets. Discrete Comput. Geom. 19, 437-445 (1998)

15. Pach, J., Tóth, G.: Erdős-Szekeres-type theorems for segments and non-crossing convex sets. Geom. Dedic. 81, 1-12 (2000)

16. Valtr, P.: A sufficient condition for the existence of large empty convex polygons. Discrete Comput. Geom. 28, 671-682 (2002)

17. Wei, X., Ding, R.: More on planar point subsets with a specified number of interior points. Math. Not. 83, 684-687 (2008) (in English). Mat. Zametki 83, 752-756 (2008) (in Russian)

18. Wei, X., Ding, R.: An Erdős-Szekeres type problem for interior points. In: Surveys on Discrete and Computational Geometry. Contemporary Mathematics, vol. 453, pp. 515-528 (2008) 